
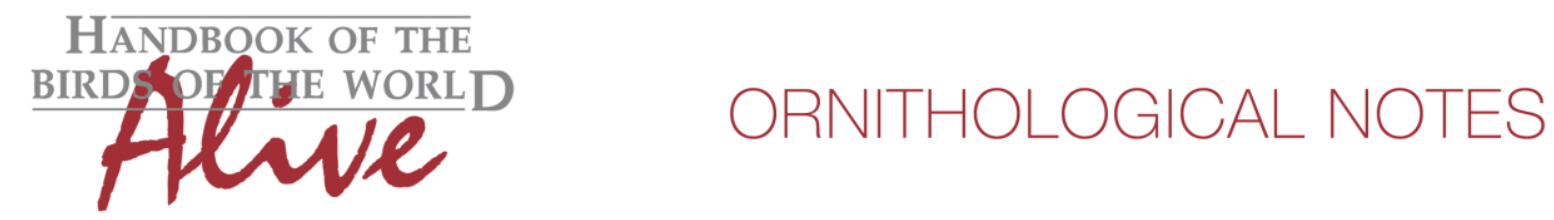

\title{
Notes on the vocalizations of Perija Antpitta (Grallaria saltuensis)
}

Peter Boesman

In the following we briefly analyze and compare voice of Perija Antpitta (Grallaria saltuensis) with the different races of Rufous Antpitta (Grallaria rufula). We also try to quantify the extent of any vocal differences using the criteria proposed by Tobias et al. (2010), as a support for taxonomic review. We have made use of sound recordings available on-line from Xeno Canto $(X C)$.

Voice of saltuensis has long been unknown, until Colombian expeditions in 2008/2009 to the area were able to record it. At present, only 4 recordings are available on XC: 3 of them are the same short trill (named by the recordists either 'call', 'short song' or 'song', the other is a long series of notes, named by the recordist 'long song'.

It is thus unclear what is the loudsong which we have to compare with the different races of Rufous Antpitta. As a thorough paper about this complex is to be expected in the near future, I will only do a 'rough' scoring in two cases:

Case 1: the long series is the loudsong (duration $5 \mathrm{~s},>25$ notes, max. note length $0.16 \mathrm{~s}$, $\mathrm{c}$. 3000Hz)

G. r. spatiator - N Colombia in Santa Marta Mts.

The song of the subspecies (ssp. spatiator in the Santa Marta Mountains is the highest pitched of all the Rufous Antpitta forms. It consists of a long ( 3 second) series of short, highpitched but descending notes, with the whole series descending.

$\rightarrow$ saltuensis differs in lower number of notes (score 2), longer max. note length (score 2) and lower frequency (score 2) -> total score 4

G. r. rufula or undescribed taxon - SW Venezuela (E \& W Táchira) and E Andes of Colombia The song in this area is made up of a series of long, clear, even whistles given at a fairly high pitch, with the whole series descending slightly.

-> saltuensis differs in higher number of notes (score 3), shorter max. note length (score 3) -> total score 6

G. r. rufula excluding previous Andes of Colombia ( $\mathrm{N}$ part of $\mathrm{W}$, and middle $\mathrm{C}$ ranges) $\mathrm{S}$ to extreme N Peru.

The songs of Rufous Antpitta (ssp. rufula) in these areas are what many birders consider the 'classic' song of the species: a short clear note followed by a rapid, mellow stutter, at a pitch a bit lower than that of the Eastern Andes form from Colombia, in all lasting about a second. It is both lower pitched and much shorter than the song from the eastern Colombia cordillera, and shorter and made up of more notes than the birds from the Cajamarca area on the other side of the Marañón River.

-> saltuensis differs in higher number of notes (score 3), longer total song length (score 3) $>$ total score 6 

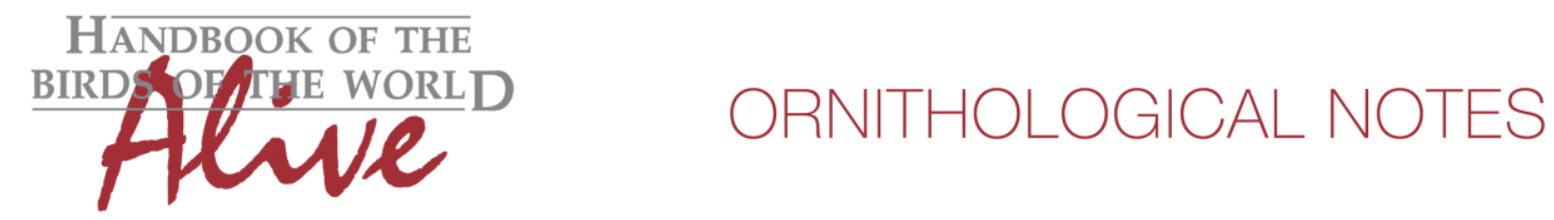

\section{G. r. cajamarcae - W Andes of Peru in S Cajamarca.}

The cajamarcae subspecies, found from northern Peru south of the Marañón River to an unknown point south of Cajamarca has a song made up of a slow series of medium-pitched, mellow notes, lasting from 3-4 seconds, and consisting of 5 to 8 or more notes. It is perhaps most like the form from the Eastern Andes of Colombia, but lower pitched, with shorter notes sounding less like whistles.

$\rightarrow$ saltuensis differs in higher number of notes (score 3), and higher pace (score 3) $\quad$-> total score 6

\section{G. r. obscura - C Andes of Peru (S to Junín).}

The song of the obscura subspecies of Rufous Antpitta, found in central Peru, is like a buzzier version of the song of the rufula subspecies. It consists of a single, fairly clear note, followed by a fast, highly modulated (buzzy) whistle, the whole vocalization lasting about a second. The second note of the song sounds like a fast police whistle.

-> saltuensis differs in longer total song length (score 3) and slower pace (score 3) -> total score 6

G. r. occabambae - SE Peru (Cuzco) SE to W Bolivia (W La Paz).

Song consists of a short, medium-pitched whistle followed by another whistle at a slightly lower pitched. Occasionally two whistles follow the first.

$\rightarrow$ saltuensis differs in higher number of notes (score 3), longer total song length (score 3) $>$ total score 6

G. r. cochabambae - C Bolivia (N Cochabamba, possibly also W to C La Paz).

Song consists of a short, medium-pitched whistle followed by another whistle at a slightly lower pitched, with a much shorter pause in between than occabambae.

$\rightarrow$ saltuensis differs in higher number of notes (score 3), longer total song length (score 3) $>$ total score 6

\section{Case 2: the short trill is the loudsong}

duration $0.42-0.5 \mathrm{~s}, 11-13$ trilled notes, pace $0.037-0.039$, min freq. $1600-1800 \mathrm{~Hz}$, max. freq. $3300-3700 \mathrm{~Hz}$

G. r. spatiator - N Colombia in Santa Marta Mts.

The song of the subspecies (ssp. spatiator in the Santa Marta Mountains is the highest pitched of all the Rufous Antpitta forms. It consists of a long (3 second) series of short, highpitched but descending notes, with the whole series descending.

$\rightarrow$ saltuensis differs in lower number of notes (score 3), shorter total length (score 3) -> total score 6

G. r. rufula or undescribed taxon - SW Venezuela (E \& W Táchira) and E Andes of Colombia The song in this area is made up of a series of long, clear, even whistles given at a fairly high pitch, with the whole series descending slightly.

$\rightarrow$ saltuensis differs in shorter max. note length (score 4) and much higher pace (score 4) -> total score 8 

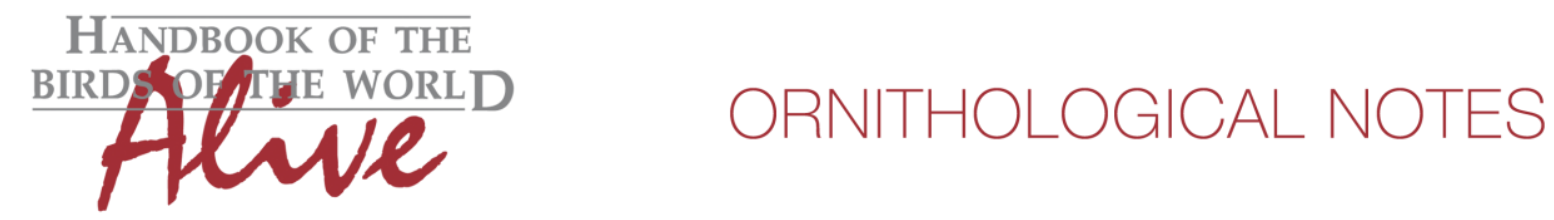

G. r. rufula excluding previous -Andes of Colombia ( $\mathrm{N}$ part of $\mathrm{W}$, and middle $\mathrm{C}$ ranges) $\mathrm{S}$ to extreme N Peru.

The songs of Rufous Antpitta (ssp. rufula) in these areas are what many birders consider the 'classic' song of the species: a short clear note followed by a rapid, mellow stutter, at a pitch a bit lower than that of the Eastern Andes form from Colombia, in all lasting about a second. It is both lower pitched and much shorter than the song from the eastern Colombia cordillera, and shorter and made up of more notes than the birds from the Cajamarca area on the other side of the Marañón River.

$\rightarrow$ saltuensis differs in higher number of notes (score 3), and higher pace (score 3) $\quad$-> total score 6

G. r. cajamarcae - W Andes of Peru in S Cajamarca.

The cajamarcae subspecies, found from northern Peru south of the Marañón River to an unknown point south of Cajamarca has a song made up of a slow series of medium-pitched, mellow notes, lasting from 3-4 seconds, and consisting of 5 to 8 or more notes. It is perhaps most like the form from the Eastern Andes of Colombia, but lower pitched, with shorter notes sounding less like whistles.

-> saltuensis differs in higher number of notes (score 3), higher pace (score 3) and shorter notes (score 3) $\rightarrow$ total score 6

G. r. obscura - C Andes of Peru (S to Junín).

The song of the obscura subspecies of Rufous Antpitta, found in central Peru, is like a buzzier version of the song of the rufula subspecies. It consists of a single, fairly clear note, followed by a fast, highly modulated (buzzy) whistle, the whole vocalization lasting about a second. The second note of the song sounds like a fast police whistle.

-> comes closest to saltuensis, but saltuensis differs in lacking introductory note with long pause before trill. Scoring based on: shorter song length (score 2), and number of notes (score 3) $\rightarrow$ total score 5

G. r. occabambae - SE Peru (Cuzco) SE to W Bolivia (W La Paz).

Song consists of a short, medium-pitched whistle followed by another whistle at a slightly lower pitched. Occasionally two whistles follow the first.

-> saltuensis differs in higher number of notes (score 3), higher pace (score 3) and shorter notes (score 3) $\rightarrow$ total score 6

G. r. cochabambae - C Bolivia (N Cochabamba, possibly also W to C La Paz).

Song consists of a short, medium-pitched whistle followed by another whistle at a slightly lower pitched, with a much shorter pause in between than occabambae.

-> saltuensis differs in higher number of notes (score 3), higher pace (score 3 ) and shorter notes (score 3) $\rightarrow$ total score 6 
It would thus seem that in either case, voice of $G$. saltuensis is very different. Although the mentioned scores should be interpreted as estimations, it is clear that we can consider these 'major' vocal differences, although at the other hand, we should not forget that this brief analysis is based on just 4 recordings of saltuensis.

This note was finalized on 30th June 2015 , using sound recordings available on-line at that moment. We would like to thank in particular the sound recordists who placed their recordings for this species on XC: Andres M. Cuervo and Fabrice Schmitt.

\section{References}

Tobias, J.A., Seddon, N., Spottiswoode, C.N., Pilgrim, J.D., Fishpool, L.D.C. \& Collar, N.J. (2010). Quantitative criteria for species delimitation. Ibis 152(4): 724-746.

\section{Recommended citation}

Boesman, P. (2016). Notes on the vocalizations of Perija Antpitta (Grallaria saltuensis). HBW Alive Ornithological Note 71. In: Handbook of the Birds of the World Alive. Lynx Edicions, Barcelona. (retrieved from http://www.hbw.com/node/931958 on 17 May 2016). 